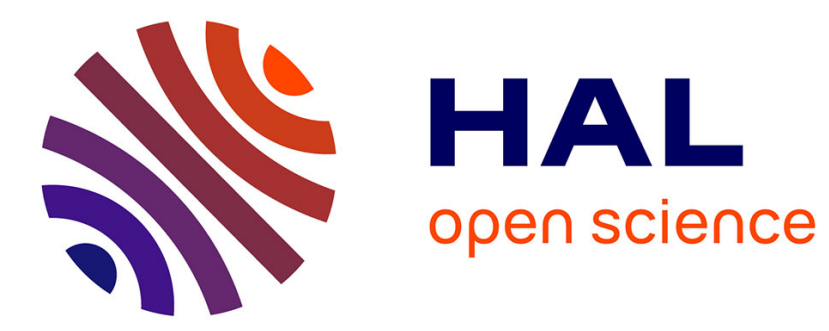

\title{
Model for the growth and the oscillation of a cavitation bubble in a spherical liquid-filled cavity enclosed in an elastic medium
}

\author{
Alexander A Doinikov, Benjamin Dollet, Philippe Marmottant
}

\section{- To cite this version:}

Alexander A Doinikov, Benjamin Dollet, Philippe Marmottant. Model for the growth and the oscillation of a cavitation bubble in a spherical liquid-filled cavity enclosed in an elastic medium. Physical Review E , 2018, 97 (1), 10.1103/PhysRevE.97.013108 . hal-01906075v2

\section{HAL Id: hal-01906075 \\ https://hal.science/hal-01906075v2}

Submitted on 8 Jul 2019

HAL is a multi-disciplinary open access archive for the deposit and dissemination of scientific research documents, whether they are published or not. The documents may come from teaching and research institutions in France or abroad, or from public or private research centers.
L'archive ouverte pluridisciplinaire HAL, est destinée au dépôt et à la diffusion de documents scientifiques de niveau recherche, publiés ou non, émanant des établissements d'enseignement et de recherche français ou étrangers, des laboratoires publics ou privés. 


\title{
Model for the growth and the oscillation of a cavitation bubble in a spherical liquid-filled cavity enclosed in an elastic medium
}

\author{
Alexander A. Doinikov, Benjamin Dollet, and Philippe Marmottant \\ LIPhy UMR 5588, CNRS/Université Grenoble-Alpes, Grenoble F-38401, France
}

(Received 20 October 2017; published 17 January 2018)

\begin{abstract}
Equations are derived that describe the growth and subsequent damped oscillation of a cavitation bubble in a liquid-filled cavity surrounded by an elastic solid. It is assumed that the nucleation and the growth of the bubble are caused by an initial negative pressure in the cavity. The liquid is treated as viscous and compressible. The obtained equations allow one to model, by numerical computation, the growth and the oscillation of the bubble in the cavity and the oscillation of the cavity surface. It is shown that the equilibrium radius reached by the growing bubble decreases when the absolute magnitude of the initial negative pressure decreases. It is also found that the natural frequency of the bubble oscillation increases with increasing bubble radius. This result is of special interest because in an unbounded liquid, the natural frequency of a bubble is known to behave oppositely, namely it decreases with increasing bubble radius.
\end{abstract}

DOI: 10.1103/PhysRevE.97.013108

\section{INTRODUCTION}

Cavitation is a well-known phenomenon [1,2]. However, most theoretical efforts in this area are devoted to cavitation in an unbounded liquid, including those that provide equations for modeling the growth and the oscillation of cavitation bubbles [3-9]. It is the assumption of an unbounded liquid that underlies the Keller-Miksis equation [10], which is used in most modern studies to model finite-amplitude bubble oscillations in a viscous compressible liquid. Cavitation in a microscopic confinement has only recently received increased attention. One of the important problems that inspires this attention is the investigation of cavitation effects that occur inside trees [11-14]. In connection with such investigations, it is desirable to have mathematical expressions to model the dynamics of a growing and oscillating cavitation bubble in a liquid-filled cavity enclosed in an elastic medium.

Evaporation processes lead to great tensile stresses inside water-filled tree conduits. Negative pressures inside the conduits can drop down to $-18.8 \mathrm{MPa}$ [12-14]. In in vitro experimental studies that imitate this process, even lower pressures are reported, of the order of $-20 \pm 2 \mathrm{MPa}$ [15-17]. High negative pressures give rise to the nucleation of cavitation bubbles whose growth causes the relaxation of tension in tree conduits. It is worth noting that, unlike cavitation bubbles in an unbounded liquid, bubbles confined in a cavity do not collapse quickly because their existence is necessary for maintaining the relaxation of negative pressure within the cavity.

In the process of growth, cavitation bubbles undergo transient oscillations and emit acoustic waves in the ultrasonic range [18]. This acoustic emission can be used to monitor the development of cavitation inside trees [19], which is a very important problem because cavitation events have a great influence on tree physiology, which can be both negative and positive [20-23].

Vincent et al. $[15,16]$ have proposed a model, based on semiqualitative considerations, that describes the dynamics of a cavitation bubble in a spherical liquid-filled cavity surrounded by an infinite elastic solid. Their model allows one to evaluate the equilibrium radius reached by the growing bubble and the frequency of the bubble oscillation but does not consider the wave propagation in the liquid and in the solid. The predictions of this model were found to be in good agreement with experimental measurements made with synthetic wood $[15,16]$.

Vincent and Marmottant [17] and Wang [24] have derived Rayleigh-Plesset-like equations that describe the finiteamplitude oscillation of a bubble in a liquid-filled cavity confined by an elastic solid. Both equations are based on a quasistatic approximation for the compressibility of the liquid and the solid, which assumes that the pressure in the cavity varies in time but is uniform in space. Both models do not consider the wave propagation in the solid.

Drysdale et al. [25] have developed a theory that describes the linear (small-amplitude) oscillation of a bubble in a cavity surrounded by an elastic solid. This theory involves acoustic waves emitted by the bubble and their propagation in the solid. Drysdale et al. [25] derived a dispersion equation that allows one to calculate the natural frequency and the attenuation coefficient of the bubble oscillation. Analysis performed by Drysdale et al. [25] predicts that the main mechanism of attenuation is related to the wave propagation in the solid.

The present study is aimed at the development of theory for nonlinear bubble dynamics in a confinement. Its specific purpose is to derive equations that describe the entire evolution of a cavitation bubble in a liquid-filled cavity enclosed in an elastic solid. The equations are intended for modeling the growth of the bubble from its nucleation until the attainment of an equilibrium radius corresponding to the relaxation of tension in the cavity, and then modeling the damped oscillation of the bubble about the attained radius. The equations of the bubble evolution are derived in Sec. II. In Sec. III, the obtained equations are applied to perform numerical simulations. To anticipate, the main results of our derivation are given by Eq. (56) in Sec. II and Eq. (A12) in Appendix A. Equation (56), like the Keller-Miksis equation [10], is an ordinary nonlinear differential equation of second order. It describes the time 

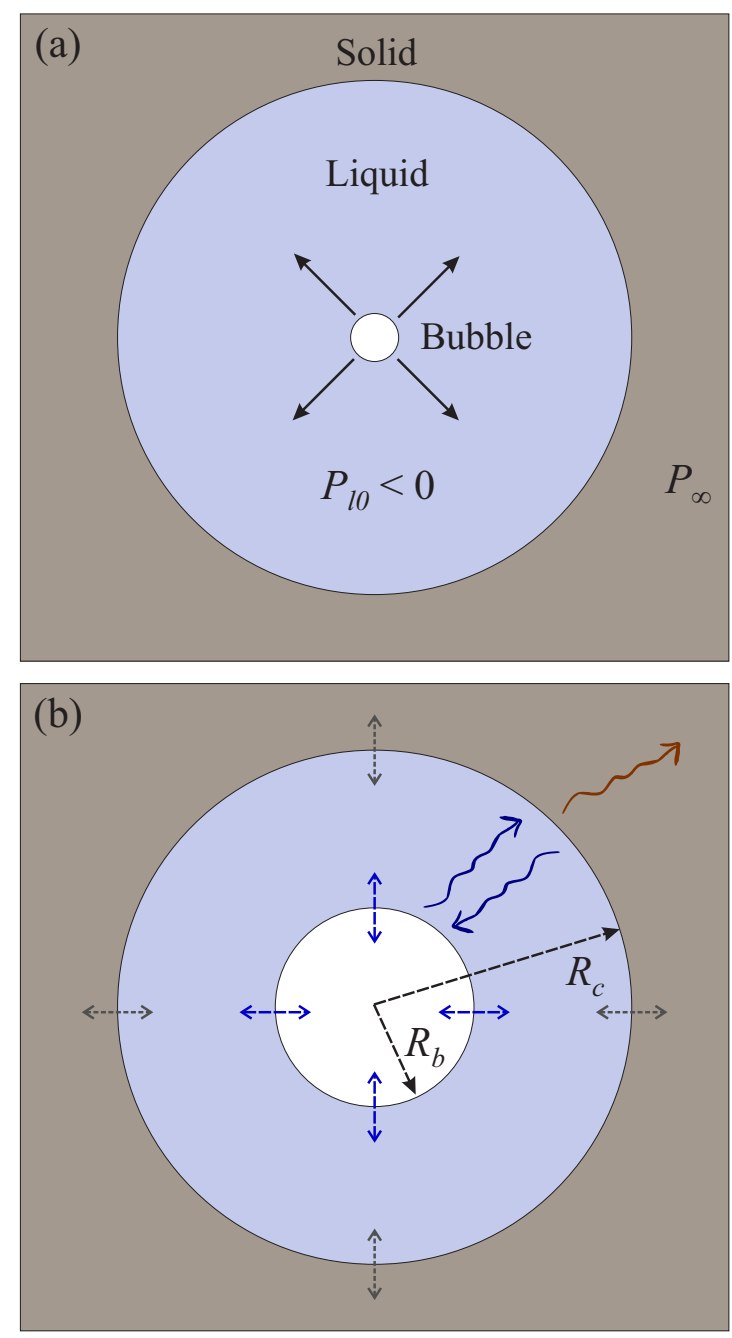

FIG. 1. Geometry of the system under study. (a) Growth of a cavitation bubble due to a negative pressure in the liquid. (b) Oscillation of the bubble around its equilibrium radius.

evolution of the bubble radius. Equation (A12) describes the elastic stress at the cavity surface, which is an important part of the equation of bubble evolution.

\section{MATHEMATICAL MODEL}

The geometry of the system under study is shown in Fig. 1. There is a spherical cavity filled with a compressible viscous liquid and surrounded by an elastic solid. A high initial negative pressure in the liquid causes the nucleation of a cavitation bubble. The bubble is growing, which allows the tension in the cavity to relax. When the bubble approaches an equilibrium radius, it undergoes a damped oscillation and emits acoustic waves, which propagate through the liquid, penetrate into the solid, and go to infinity.

\section{A. Basic equations \\ 1. Liquid}

We assume that the liquid is viscous and compressible. The motion of a viscous and compressible liquid obeys the equation of continuity and the Navier-Stokes equation [26],

$$
\begin{gathered}
\frac{\partial \rho_{l}}{\partial t}+\nabla \cdot\left(\rho_{l} \boldsymbol{v}\right)=0, \\
\rho_{l} \frac{\partial \boldsymbol{v}}{\partial t}+\rho_{l}(\boldsymbol{v} \cdot \nabla) \boldsymbol{v} \\
=-\nabla p+\eta_{l} \Delta \boldsymbol{v}+\left(\zeta_{l}+\frac{1}{3} \eta_{l}\right) \nabla(\nabla \cdot \boldsymbol{v}),
\end{gathered}
$$

where $v, \rho_{l}, p, \eta_{l}$, and $\zeta_{l}$ are the velocity, the density, the pressure, the shear viscosity, and the bulk viscosity, respectively.

In the case under consideration, the liquid flow is spherically symmetric and hence irrotational [26]. In an irrotational flow, the vector velocity, $\boldsymbol{v}$, can be written in terms of a velocity potential, $\varphi$, as

$$
\boldsymbol{v}=v(r, t) \boldsymbol{e}_{r}=\nabla \varphi(r, t)=\frac{\partial \varphi}{\partial r} \boldsymbol{e}_{r},
$$

where $\boldsymbol{e}_{r}$ is the unit vector along the $r$ coordinate. On substitution of Eq. (3), Eqs. (1) and (2) take the form

$$
\begin{gathered}
\rho_{l} \Delta \varphi+\frac{\partial \rho_{l}}{\partial t}+\frac{\partial \varphi}{\partial r} \frac{\partial \rho_{l}}{\partial r}=0 \\
\rho_{l} \frac{\partial}{\partial r}\left[\frac{\partial \varphi}{\partial t}+\frac{1}{2}\left(\frac{\partial \varphi}{\partial r}\right)^{2}\right]=\frac{\partial}{\partial r}\left[-p+\left(\zeta_{l}+\frac{4}{3} \eta_{l}\right) \Delta \varphi\right]
\end{gathered}
$$

In what follows, we will need the normal stress in the liquid. It is given by [26]

$$
\sigma_{r r}=-p+2 \eta_{l} \frac{\partial^{2} \varphi}{\partial r^{2}}+\left(\zeta_{l}-\frac{2}{3} \eta_{l}\right) \Delta \varphi .
$$

\section{Solid}

The motion of the solid is described by the Navier equation [27],

$$
\rho_{s} \frac{\partial^{2} \boldsymbol{u}}{\partial t^{2}}=\mu \Delta \boldsymbol{u}+(\lambda+\mu) \nabla(\nabla \cdot \boldsymbol{u}),
$$

where $\boldsymbol{u}$ is the displacement vector, $\partial \boldsymbol{u} / \partial t$ is the velocity in the solid, $\rho_{s}$ is the density of the solid, and $\lambda$ and $\mu$ are the Lamé coefficients. In view of spherical symmetry, $\boldsymbol{u}$ can be written in terms of a potential $\varphi_{s}$ as

$$
\boldsymbol{u}=u(r, t) \boldsymbol{e}_{r}=\nabla \varphi_{s}(r, t)=\frac{\partial \varphi_{s}}{\partial r} \boldsymbol{e}_{r} .
$$

Substitution of Eq. (8) into Eq. (7) yields

$$
\nabla\left(\Delta \varphi_{s}-\frac{1}{c_{s}^{2}} \frac{\partial^{2} \varphi_{s}}{\partial t^{2}}\right)=0
$$

where $c_{s}$ is the longitudinal wave speed, given by [27]

$$
c_{s}=\sqrt{\frac{\lambda+2 \mu}{\rho_{s}}} .
$$


From Eq. (9), one has

$$
\Delta \varphi_{s}-\frac{1}{c_{s}^{2}} \frac{\partial^{2} \varphi_{s}}{\partial t^{2}}=a
$$

where $a$ is, in general, a constant or a time function. In our case, a solution to Eq. (11) is written as

$$
\varphi_{s}(r, t)=\frac{a r^{2}}{6}-\frac{s\left(t-r / c_{s}\right)}{r},
$$

where the first term is the solution of the equation $\Delta \varphi_{s}=a$ and the second term is the solution of the wave equation $\Delta \varphi_{s}-$ $c_{s}^{-2} \partial^{2} \varphi_{s} / \partial t^{2}=0$. Note that $a$ must be a constant in order that Eq. (12) satisfies Eq. (11).

It is important to emphasize the difference between Eq. (12) and the solution used in the linear analysis of Drysdale et al. [25]; see Eq. (14) in their paper. Drysdale et al. [25] assume that the system is under the same ambient pressure all the time and there is no initial strain in the solid. Based on this assumption, they consider only wave processes that occur in the system. In the present study, we assume that the liquid is initially under a high negative pressure, whereas the pressure in the solid at infinity is atmospheric, which causes a strain distribution in the solid that compensates the pressure difference prior to bubble nucleation. In the process of the bubble growth, the ambient liquid pressure changes and the strain in the solid relaxes. The description of the relaxation process requires the inclusion of the first term in Eq. (12), while the second term describes an outgoing acoustic wave.

From Eq. (12), the displacement field is expressed as

$$
u=\frac{\partial \varphi_{s}}{\partial r}=\frac{a r}{3}+\frac{s\left(t-r / c_{s}\right)}{r^{2}}+\frac{s^{\prime}\left(t-r / c_{s}\right)}{c_{s} r},
$$

where the prime denotes the derivative with respect to the argument in brackets.

The normal stress in the solid is given by [27]

$$
\tau_{r r}=\lambda \nabla \cdot \boldsymbol{u}+2 \mu \frac{\partial u}{\partial r} .
$$

Substitution of Eq. (13) into Eq. (14) yields

$$
\begin{aligned}
\tau_{r r}= & \left(\lambda+\frac{2 \mu}{3}\right) a-4 \mu\left[\frac{s\left(t-r / c_{s}\right)}{r^{3}}+\frac{s^{\prime}\left(t-r / c_{s}\right)}{c_{s} r^{2}}\right] \\
& -\frac{(\lambda+2 \mu) s^{\prime \prime}\left(t-r / c_{s}\right)}{c_{s}^{2} r} .
\end{aligned}
$$

We assume that for $r \rightarrow \infty, \tau_{r r} \rightarrow-P_{\infty}$, where $P_{\infty}$ is a constant (atmospheric) pressure at infinity. From this condition, we find

$$
a=-\frac{3 P_{\infty}}{3 \lambda+2 \mu} .
$$

\section{Boundary conditions}

The boundary conditions assume the continuity of velocity and normal stress at the bubble surface and at the liquid-solid interface, which gives

$$
\begin{gathered}
\frac{\partial \varphi}{\partial r}=\dot{R}_{b} \quad \text { at } r=R_{b}, \\
P_{b}=\frac{2 \sigma_{l}}{R_{b}}-\sigma_{r r} \quad \text { at } r=R_{b},
\end{gathered}
$$

$$
\begin{gathered}
\frac{\partial \varphi}{\partial r}=\frac{\partial u}{\partial t}=\dot{R}_{c} \quad \text { at } r=R_{c}, \\
\sigma_{r r}=\tau_{r r} \quad \text { at } r=R_{c},
\end{gathered}
$$

where $R_{b}$ is the time-varying radius of the bubble, the overdot denotes the time derivative, $P_{b}$ is the pressure within the bubble, $\sigma_{l}$ is the surface tension, and $R_{c}$ is the time-varying radius of the cavity. Equations (17) and (19) are known as the kinematic boundary conditions, and Eqs. (18) and (20), as the dynamic boundary conditions.

\section{B. Solutions for an incompressible liquid}

We first find solutions treating the liquid as incompressible. We will lean upon them when deriving solutions for a compressible liquid.

In an incompressible liquid, $\rho_{l}$ is constant. As a consequence, Eqs. (4) and (5) give

$$
\begin{gathered}
\varphi=-\frac{A(t)}{r} \\
p=B(t)-\rho_{l}\left[\frac{\partial \varphi}{\partial t}+\frac{1}{2}\left(\frac{\partial \varphi}{\partial r}\right)^{2}\right],
\end{gathered}
$$

where $A$ and $B$ are unknown functions. Substitution of Eq. (21) into Eq. (17) yields

$$
A(t)=R_{b}^{2} \dot{R}_{b}
$$

Substituting Eqs. (21) and (23) into Eq. (22), one obtains the pressure field,

$$
p(r, t)=B(t)+\frac{\rho_{l}\left(R_{b}^{2} \ddot{R}_{b}+2 R_{b} \dot{R}_{b}^{2}\right)}{r}-\frac{\rho_{l} R_{b}^{4} \dot{R}_{b}^{2}}{2 r^{4}} .
$$

Substitution of Eqs. (21), (23), and (24) into Eq. (6) yields the normal stress in the liquid,

$$
\begin{aligned}
\sigma_{r r}(r, t)= & -B(t)-\frac{\rho_{l}\left(R_{b}^{2} \ddot{R}_{b}+2 R_{b} \dot{R}_{b}^{2}\right)}{r} \\
& +\frac{\rho_{l} R_{b}^{4} \dot{R}_{b}^{2}}{2 r^{4}}-\frac{4 \eta_{l} R_{b}^{2} \dot{R}_{b}}{r^{3}} .
\end{aligned}
$$

On substitution of Eq. (25) into Eq. (18), one has

$$
\rho_{l} R_{b} \ddot{R}_{b}+\frac{3}{2} \rho_{l} \dot{R}_{b}^{2}+\frac{4 \eta_{l} \dot{R}_{b}}{R_{b}}+\frac{2 \sigma_{l}}{R_{b}}+B(t)=P_{b} .
$$

$B(t)$ in this equation serves as a driving pressure that, along with $P_{b}$, drives the motion of the bubble wall. If $P_{b}=0$, as in our case actually, then $B(t)$ is the only source of the bubble evolution.

To find $B(t)$, we use Eq. (20). Substitution of Eq. (25) into Eq. (20) yields

$$
\begin{aligned}
B(t)= & -\frac{\rho_{l}\left(R_{b}^{2} \ddot{R}_{b}+2 R_{b} \dot{R}_{b}^{2}\right)}{R_{c}}+\frac{\rho_{l} R_{b}^{4} \dot{R}_{b}^{2}}{2 R_{c}^{4}} \\
& -\frac{4 \eta_{l} R_{b}^{2} \dot{R}_{b}}{R_{c}^{3}}-\tau_{r r}\left(R_{c}, t\right) .
\end{aligned}
$$

This equation reveals that $B(t)$ is a difference between the normal stress in the liquid on the inner side of the cavity surface 
and the normal stress in the solid on the outer side of the cavity surface.

Substitution of Eq. (27) into Eq. (26) yields

$$
\begin{gathered}
\rho_{l} R_{b} \ddot{R}_{b}\left(1-\frac{R_{b}}{R_{c}}\right)+\rho_{l} \dot{R}_{b}^{2}\left(\frac{3}{2}-\frac{2 R_{b}}{R_{c}}+\frac{R_{b}^{4}}{2 R_{c}^{4}}\right) \\
+\frac{4 \eta_{l} \dot{R}_{b}}{R_{b}}\left(1-\frac{R_{b}^{3}}{R_{c}^{3}}\right)+\frac{2 \sigma_{l}}{R_{b}}=\tau_{r r}\left(R_{c}, t\right)+P_{b}
\end{gathered}
$$

The left-hand side of this equation is in agreement with the equation derived by Wang [24]; see Eq. (2.19) in his paper.

To complete the derivation, it is necessary to get an expression for $\tau_{r r}\left(R_{c}, t\right)$. In order not to overburden the text, the expression for $\tau_{r r}\left(R_{c}, t\right)$, accurate up to $1 / c_{s}^{3}$, is calculated in Appendix A.

Equation (28) should be supplemented with a relation between $R_{b}$ and $R_{c}$. To this end, we apply the mass conservation law to the liquid. Since the liquid is assumed incompressible in this subsection, we can write

$$
R_{c}^{3}-R_{b}^{3}=R_{c 0}^{3}-R_{b 0}^{3},
$$

where $R_{c 0}=R_{c}(0)$ and $R_{b 0}=R_{b}(0)$. The value of $R_{c 0}$ can be set arbitrarily. To trigger the motion of the bubble wall in numerical simulations, the initial bubble radius should be set from the condition

$$
R_{b 0}>\frac{2 \sigma_{l}}{P_{b}-P_{l 0}}
$$

where $P_{l 0}$ denotes the initial (negative) pressure in the liquid.

To sum up, the evolution of the bubble radius in the case of an incompressible liquid is calculated by Eq. (28) using Eqs. (29) and (30) and Eq. (A12) for $\tau_{r r}\left(R_{c}, t\right)$.

\section{Solutions for a compressible liquid}

The equations for the solid derived above and in Appendix A remain valid and will be used in calculations that follow.

\section{Equation for $R_{b}(t)$ with compressibility corrections}

If the liquid is treated as slightly compressible, i.e., the Mach number is much smaller than unity, we can apply the approximation proposed by Keller and Miksis [10]. Following their approach, we omit the bulk viscosity $\zeta_{l}$ in Eq. (5) and neglect change in the liquid density $\rho_{l}$. As a result, the integration of Eq. (5) gives

$$
p(r, t)=C(t)-\rho_{l}\left[\frac{\partial \varphi}{\partial t}+\frac{1}{2}\left(\frac{\partial \varphi}{\partial r}\right)^{2}\right]+\frac{4 \eta_{l}}{3} \Delta \varphi .
$$

We can set $C(t)=0$ without the loss of generality. The reason is that $\varphi$ is defined with accuracy up to an arbitrary time function and hence we can include a time function in $\varphi$ to eliminate $C(t)$ in Eq. (31).

In Eq. (4), we omit nonlinear terms assuming that relative variations of the liquid density due to acoustic waves are small.
This leads to the wave equation for $\varphi$,

$$
\Delta \varphi-\frac{1}{c^{2}} \frac{\partial^{2} \varphi}{\partial t^{2}}=0
$$

where $c$ is the speed of sound in the liquid. A solution to Eq. (32) is written as

$$
\varphi(r, t)=-\frac{f(t-r / c)}{r}-\frac{g(t+r / c)}{r},
$$

where the first term describes the acoustic emission of the bubble and the second term describes reflections from the cavity surface. It should be pointed out that solution (33) does not impose any restrictions on the number of reflections of acoustic waves between the bubble wall and the cavity wall; i.e., it accounts for multiple reflections.

Substitution of Eq. (33) into Eq. (17) yields

$$
\begin{aligned}
\left(\frac{\partial \varphi}{\partial r}\right)_{R_{b}} & =-\frac{\varphi\left(R_{b}, t\right)}{R_{b}}+\frac{f^{\prime}\left(t-R_{b} / c\right)}{c R_{b}}-\frac{g^{\prime}\left(t+R_{b} / c\right)}{c R_{b}} \\
& =\dot{R}_{b}
\end{aligned}
$$

Setting $\zeta_{l}=0$ in Eq. (6) and substituting Eq. (31) into it, one obtains

$$
\sigma_{r r}(r, t)=\rho_{l}\left[\frac{\partial \varphi}{\partial t}+\frac{1}{2}\left(\frac{\partial \varphi}{\partial r}\right)^{2}\right]-\frac{4 \eta_{l}}{r} \frac{\partial \varphi}{\partial r} .
$$

Substituting Eq. (35) into Eq. (18) and using Eq. (34), one gets

$$
P_{b}=-\rho_{l}\left(\frac{\partial \varphi}{\partial t}\right)_{R_{b}}-\frac{\rho_{l} \dot{R}_{b}^{2}}{2}+\frac{4 \eta_{l} \dot{R}_{b}}{R_{b}}+\frac{2 \sigma_{l}}{R_{b}} .
$$

The next step is the calculation of $(\partial \varphi / \partial t)_{R_{b}}$. From Eq. (33) it follows that

$$
\left(\frac{\partial \varphi}{\partial t}\right)_{R_{b}}=-\frac{f^{\prime}\left(t-R_{b} / c\right)}{R_{b}}-\frac{g^{\prime}\left(t+R_{b} / c\right)}{R_{b}} .
$$

Eliminating $f^{\prime}\left(t-R_{b} / c\right)$ from Eq. (37) by Eq. (34), one obtains

$$
\left(\frac{\partial \varphi}{\partial t}\right)_{R_{b}}=-\frac{2 g^{\prime}\left(t+R_{b} / c\right)}{R_{b}}-c \dot{R}_{b}-\frac{c \varphi\left(R_{b}, t\right)}{R_{b}} .
$$

Substitution of Eq. (38) into Eq. (36) yields

$$
\begin{aligned}
\varphi\left(R_{b}, t\right)= & \frac{R_{b} P_{b}}{c \rho_{l}}-R_{b} \dot{R}_{b}+\frac{R_{b} \dot{R}_{b}^{2}}{2 c}-\frac{4 \eta_{l} \dot{R}_{b}}{c \rho_{l}} \\
& -\frac{2 \sigma_{l}}{c \rho_{l}}-\frac{2}{c} g^{\prime}\left(t+R_{b} / c\right) .
\end{aligned}
$$

Calculation of the full time derivative of both sides of Eq. (39) gives

$$
\begin{aligned}
\left(\frac{\partial \varphi}{\partial t}\right)_{R_{b}}= & R_{b} \ddot{R}_{b}\left(\frac{\dot{R}_{b}}{c}-\frac{4 \eta_{l}}{c \rho_{l} R_{b}}-1\right)+\dot{R}_{b}^{2}\left(\frac{\dot{R}_{b}}{2 c}-2\right) \\
& +\frac{\dot{R}_{b} P_{b}+R_{b} \dot{P}_{b}}{c \rho_{l}}-\frac{2}{c}\left(1+\frac{\dot{R}_{b}}{c}\right) g^{\prime \prime}\left(t+R_{b} / c\right) .
\end{aligned}
$$


Substituting Eq. (40) into Eq. (36), one obtains

$$
\begin{aligned}
\rho_{l} R_{b} \ddot{R}_{b}\left(1-\frac{\dot{R}_{b}}{c}+\frac{4 \eta_{l}}{c \rho_{l} R_{b}}\right)+\rho_{l} \dot{R}_{b}^{2}\left(\frac{3}{2}-\frac{\dot{R}_{b}}{2 c}\right) \\
+\frac{4 \eta_{l} \dot{R}_{b}}{R_{b}}+\frac{2 \sigma_{l}}{R_{b}}+\frac{2 \rho_{l}}{c}\left(1+\frac{\dot{R}_{b}}{c}\right) g^{\prime \prime}\left(t+R_{b} / c\right) \\
=P_{b}\left(1+\frac{\dot{R}_{b}}{c}\right)+\frac{R_{b} \dot{P}_{b}}{c} .
\end{aligned}
$$

Like $B(t)$ in Eq. (26), the function $\left(2 \rho_{l} / c\right) g^{\prime \prime}\left(t+R_{b} / c\right)$ in Eq. (41) plays the role of a driving pressure, which is a difference between the pressure inside the bubble and the pressure in the liquid at the bubble surface. To find this function, Eqs. (19) and (20) are used.

From Eq. (19), one has

$$
\begin{aligned}
\left(\frac{\partial \varphi}{\partial r}\right)_{R_{c}} & =-\frac{\varphi\left(R_{c}, t\right)}{R_{c}}+\frac{f^{\prime}\left(t-R_{c} / c\right)}{c R_{c}}-\frac{g^{\prime}\left(t+R_{c} / c\right)}{c R_{c}} \\
& =\left(\frac{\partial u}{\partial t}\right)_{R_{c}}=\frac{s^{\prime}\left(t-R_{c} / c_{L}\right)}{R_{c}^{2}}+\frac{s^{\prime \prime}\left(t-R_{c} / c_{L}\right)}{c_{L} R_{c}} \\
& =\dot{R}_{c}
\end{aligned}
$$

From Eqs. (35) and (42), one gets

$$
\sigma_{r r}\left(R_{c}, t\right)=\rho_{l}\left(\frac{\partial \varphi}{\partial t}\right)_{R_{c}}+\frac{\rho_{l} \dot{R}_{c}^{2}}{2}-\frac{4 \eta_{l} \dot{R}_{c}}{R_{c}} .
$$

Substitution of Eq. (43) into Eq. (20) yields

$$
\left(\frac{\partial \varphi}{\partial t}\right)_{R_{c}}=\frac{\tau_{r r}\left(R_{c}, t\right)}{\rho_{l}}-\frac{\dot{R}_{c}^{2}}{2}+\frac{4 \eta_{l} \dot{R}_{c}}{\rho_{l} R_{c}} .
$$

On the other hand, Eq. (33) gives

$$
\left(\frac{\partial \varphi}{\partial t}\right)_{R_{c}}=-\frac{f^{\prime}\left(t-R_{c} / c\right)}{R_{c}}-\frac{g^{\prime}\left(t+R_{c} / c\right)}{R_{c}} .
$$

Eliminating $f^{\prime}\left(t-R_{c} / c\right)$ from Eq. (45) by Eq. (42), one obtains

$$
\left(\frac{\partial \varphi}{\partial t}\right)_{R_{c}}=-\frac{2 g^{\prime}\left(t+R_{c} / c\right)}{R_{c}}-c \dot{R}_{c}-\frac{c \varphi\left(R_{c}, t\right)}{R_{c}} .
$$

Substitution of Eq. (46) into Eq. (44) yields

$$
\begin{aligned}
\varphi\left(R_{c}, t\right)= & \frac{R_{c} \dot{R}_{c}^{2}}{2 c}-R_{c} \dot{R}_{c}-\frac{4 \eta_{l} \dot{R}_{c}}{c \rho_{l}}-\frac{R_{c} \tau_{r r}\left(R_{c}, t\right)}{c \rho_{l}} \\
& -\frac{2}{c} g^{\prime}\left(t+R_{c} / c\right) .
\end{aligned}
$$

Calculation of the full time derivative of both sides of Eq. (47) gives

$$
\begin{aligned}
\left(\frac{\partial \varphi}{\partial t}\right)_{R_{c}}= & R_{c} \ddot{R}_{c}\left(\frac{\dot{R}_{c}}{c}-1-\frac{4 \eta_{l}}{c \rho_{l} R_{c}}\right)-\dot{R}_{c}^{2}\left(2-\frac{\dot{R}_{c}}{2 c}\right) \\
& -\frac{\dot{R}_{c} \tau_{r r}\left(R_{c}, t\right)}{c \rho_{l}}-\frac{R_{c}}{c \rho_{l}} \frac{d \tau_{r r}\left(R_{c}, t\right)}{d t} \\
& -\frac{2}{c}\left(1+\frac{\dot{R}_{c}}{c}\right) g^{\prime \prime}\left(t+R_{c} / c\right) .
\end{aligned}
$$

Substitution of Eq. (48) into Eq. (44) yields

$$
\begin{aligned}
& \frac{2 \rho_{l}}{c}\left(1+\frac{\dot{R}_{c}}{c}\right) g^{\prime \prime}\left(t+R_{c} c\right) \\
& =-\rho_{l} R_{c} \ddot{R}_{c}\left(1-\frac{\dot{R}_{c}}{c}+\frac{4 \eta_{l}}{c \rho_{l} R_{c}}\right)-\rho_{l} \dot{R}_{c}^{2}\left(\frac{3}{2}-\frac{\dot{R}_{c}}{2 c}\right) \\
& \quad-\frac{4 \eta_{l} \dot{R}_{c}}{R_{c}}-\tau_{r r}\left(R_{c}, t\right)\left(1+\frac{\dot{R}_{c}}{c}\right)-\frac{R_{c}}{c} \frac{d \tau_{r r}\left(R_{c}, t\right)}{d t} .
\end{aligned}
$$

In view of the different arguments of the function $g^{\prime \prime}$, Eq. (49) cannot be substituted immediately into Eq. (41). To resolve this problem, we assume that the effect of the time delays $R_{b, c} / c$ on the behavior of the function $g^{\prime \prime}$ is small and hence this function can be expanded into a Taylor series in terms of $R_{b, c} / c$. Then, we can represent Eqs. (41) and (49) as follows:

$$
\begin{aligned}
& \frac{2 \rho_{l}}{c} g^{\prime \prime}\left(t+R_{b} / c\right) \approx \frac{2 \rho_{l}}{c}\left(g^{\prime \prime}(t)+\frac{R_{b}}{c} g^{\prime \prime \prime}(t)\right)=E_{b}, \\
& \frac{2 \rho_{l}}{c} g^{\prime \prime}\left(t+R_{c} / c\right) \approx \frac{2 \rho_{l}}{c}\left(g^{\prime \prime}(t)+\frac{R_{c}}{c} g^{\prime \prime \prime}(t)\right)=E_{c},
\end{aligned}
$$

where

$$
\begin{aligned}
E_{b}= & P_{b}+\frac{R_{b} \dot{P}_{b}}{c}-\rho_{l} R_{b} \ddot{R}_{b}\left(1-\frac{2 \dot{R}_{b}}{c}+\frac{4 \eta_{l}}{c \rho_{l} R_{b}}\right) \\
& -\rho_{l} \dot{R}_{b}^{2}\left(\frac{3}{2}-\frac{2 \dot{R}_{b}}{c}\right)-\frac{4 \eta_{l} \dot{R}_{b}+2 \sigma_{l}}{R_{b}}\left(1-\frac{\dot{R}_{b}}{c}\right), \\
E_{c}= & -\rho_{l} R_{c} \ddot{R}_{c}\left(1-\frac{2 \dot{R}_{c}}{c}+\frac{4 \eta_{l}}{c \rho_{l} R_{c}}\right)-\rho_{l} \dot{R}_{c}^{2}\left(\frac{3}{2}-\frac{2 \dot{R}_{c}}{c}\right) \\
& -\frac{4 \eta_{l} \dot{R}_{c}}{R_{c}}\left(1-\frac{\dot{R}_{c}}{c}\right)-\tau_{r r}\left(R_{c}, t\right)-\frac{R_{c}}{c} \frac{d \tau_{r r}\left(R_{c}, t\right)}{d t} .
\end{aligned}
$$

From Eqs. (50) and (51) it follows that

$$
E_{b}+\frac{R_{c}-R_{b}}{c} \frac{2 \rho_{l}}{c} g^{\prime \prime \prime}(t)=E_{c} .
$$

The factor $1 / c$ in front of the function $\left(2 \rho_{l} / c\right) g / / /(t)$ allows us to calculate this function neglecting compressibility corrections, i.e., in the limit $c \rightarrow \infty$. Equation (50) shows that for $c \rightarrow \infty,\left(2 \rho_{l} / c\right) g^{\prime \prime}(t)$ is approximated by Eq. (52) with $c \rightarrow \infty$. Therefore, Eq. (54) can be recast to

$$
E_{b}+\frac{R_{c}-R_{b}}{c} \dot{E}_{b}^{\mathrm{inc}}=E_{c},
$$

where $E_{b}^{\text {inc }}$ denotes Eq. (52) at $c \rightarrow \infty$, i.e., in the limit of an incompressible liquid.

The differentiation of $E_{b}^{\text {inc }}$ leads to the appearance of the third derivative, $\dddot{R}_{b}$. To eliminate it, the solution for an incompressible liquid can be used, Eq. (28), which allows one to express $\dddot{R}_{b}$ in terms of derivatives of lower order. As a result of all these operations, after cumbersome but straightforward 
calculations, we obtain the following final equation:

$$
\begin{aligned}
& \rho_{l} R_{b} \ddot{R}_{b}\left(1-\frac{\dot{R}_{b} R_{b}^{3}}{c R_{c}^{3}}-\frac{\dot{R}_{c} R_{b}}{c R_{c}}+\frac{4 \eta_{l} R_{b}}{c \rho_{l} R_{c}^{2}}\right) \\
& \quad+\rho_{l} \dot{R}_{b}^{2}\left[\frac{3}{2}-\frac{2 \dot{R}_{b} R_{b}^{3}}{c R_{c}^{3}}-\frac{2 \dot{R}_{c} R_{b}}{c R_{c}}\left(1-\frac{R_{b}^{3}}{R_{c}^{3}}\right)+\frac{4 \eta_{l}}{c \rho_{l} R_{b}}\left(1+\frac{2 R_{b}^{2}}{R_{c}^{2}}\right)\right]+\frac{4 \eta_{l} \dot{R}_{b}}{R_{b}}\left(1-\frac{\dot{R}_{b}}{c}-\frac{3 \dot{R}_{c} R_{b}^{3}}{c R_{c}^{3}}\right)+\frac{2 \sigma_{l}}{R_{b}}-P_{b} \\
&=\rho_{l} R_{c} \ddot{R}_{c}\left(1-\frac{2 \dot{R}_{c}}{c}+\frac{4 \eta_{l}}{c \rho_{l} R_{c}}\right)+\rho_{l} \dot{R}_{c}^{2}\left(\frac{3}{2}-\frac{2 \dot{R}_{c}}{c}\right)+\frac{4 \eta_{l} \dot{R}_{c}}{R_{c}}\left(1-\frac{\dot{R}_{c}}{c}\right)+\tau_{r r}\left(R_{c}, t\right) .
\end{aligned}
$$

For reference, Appendix B provides equations for $\dddot{R}_{b}$ and $\dot{E}_{b}^{\text {inc }}$, which were used for the derivation of Eq. (56).

\section{Relation between $R_{b}$ and $R_{c}$}

Equation (56) should be supplemented with a relation between $R_{b}$ and $R_{c}$. To this end, we apply Eqs. (34) and (42).

Expanding the functions $f$ and $g$ in Eq. (34) into a Taylor series, one obtains

$$
f(t)+g(t)=R_{b}^{2} \dot{R}_{b}+O\left(\frac{1}{c^{2}}\right) .
$$

Doing the same with Eq. (42), one has

$$
f(t)+g(t)=R_{c}^{2} \dot{R}_{c}+O\left(\frac{1}{c^{2}}\right) .
$$

These results reveal that the relation

$$
R_{b}^{2} \dot{R}_{b}=R_{c}^{2} \dot{R}_{c}
$$

is valid up to the order $1 / c$. This accuracy corresponds to the accuracy of Eq. (56) with respect to the liquid compressibility. Therefore, Eq. (59) can be used along with Eq. (56). It should be mentioned that the application of the mass conservation law to the liquid also confirms that a deviation from Eq. (59) is only of the order $1 / c^{2}$.

\section{Linearized equations}

We will see in Sec. III that the oscillation of the cavity surface is weak. When the growing bubble reaches a terminal equilibrium radius, its oscillation becomes weak as well. Therefore, implying this stage, we can linearize Eq. (56). We assume $P_{b}=0$ in this calculation because the main purpose of the linearization is a comparison with the results of Drysdale et al. [25], where the bubble interior was assumed to be vacuum.

We assume that

$$
\begin{aligned}
& R_{b}(t)=R_{\mathrm{be}}+x_{b}(t),\left|x_{b}\right| \ll R_{\mathrm{be}}, \\
& R_{c}(t)=R_{\mathrm{ce}}+x_{c}(t),\left|x_{c}\right| \ll R_{\mathrm{ce}},
\end{aligned}
$$

where $R_{\mathrm{be}}$ and $R_{\mathrm{ce}}$ are the final equilibrium radii of the bubble and the cavity. Substituting these equations into Eq. (56) and keeping only time-independent terms, one obtains

$$
\frac{2 \sigma_{l}}{R_{\mathrm{be}}}+\left(4 \mu-a c_{s}^{2} \rho_{s}\right)\left(1-\frac{R_{c 0}}{R_{\mathrm{ce}}}\right)+\frac{R_{c 0} P_{l 0}}{R_{\mathrm{ce}}}=0 .
$$

This equation should be supplemented with the equation

$$
R_{\mathrm{ce}}^{3}-R_{\mathrm{be}}^{3}=R_{c 0}^{3}-R_{b 0}^{3},
$$

which, like Eq. (59), is correct up to the order $1 / c$. Solving simultaneously Eqs. (62) and (63) gives the values of $R_{\text {be }}$ and $R_{\text {ce }}$.

Keeping only linear time-varying terms in Eq. (56), one obtains

$$
\begin{aligned}
& \rho_{l} R_{\mathrm{be}} \ddot{x}_{b}\left(1+\frac{4 \eta_{l} R_{\mathrm{be}}}{c \rho_{l} R_{\mathrm{ce}}^{2}}\right)+\frac{4 \eta_{l} \dot{x}_{b}}{R_{\mathrm{be}}}-\frac{2 \sigma_{l} x_{b}}{R_{\mathrm{be}}^{2}} \\
& =\left(a c_{s}^{2} \rho_{s}+P_{l 0}-4 \mu\right) \frac{R_{c 0} x_{c}}{R_{\mathrm{ce}}^{2}}+R_{\mathrm{ce}} \ddot{x}_{c}\left(\rho_{l}-\rho_{s}+\frac{4 \eta_{l}}{c R_{\mathrm{ce}}}-\frac{4 \eta_{l} \rho_{s}\left(R_{\mathrm{ce}}^{3}-R_{\mathrm{be}}^{3}\right)}{c_{s} \rho_{l} R_{\mathrm{ce}} R_{\mathrm{be}}^{2}\left[R_{\mathrm{ce}}-R_{\mathrm{be}}\left(1-\rho_{s} / \rho_{l}\right)\right]}\right) \\
& \quad+\frac{4 \eta_{l} \dot{x}_{c}}{R_{\mathrm{ce}}}+\frac{\dot{x}_{c} \rho_{s}}{c_{s} \rho_{l}\left[R_{\mathrm{ce}}-R_{\mathrm{be}}\left(1-\rho_{s} / \rho_{l}\right)\right]}\left(\left(a c_{s}^{2} \rho_{s}+P_{l 0}-4 \mu\right) \frac{R_{b e} R_{c 0}}{R_{\mathrm{ce}}}+\frac{2 \sigma_{l} R_{\mathrm{ce}}^{3}}{R_{\mathrm{be}}^{3}}\right) .
\end{aligned}
$$

To solve this equation, we assume that

$$
x_{b}=a_{b} e^{-i \omega t}, \quad x_{c}=a_{c} e^{-i \omega t} .
$$

Substituting Eqs. (65) into Eq. (64), using the linearized Eq. (59) to express $a_{c}$ in terms of $a_{b}$, and then removing $a_{b}$, we obtain the following equation:

$$
\omega^{2}+2 i \alpha \omega-\omega_{0}^{2}=0
$$


in which $\omega$ is a sought quantity and the other quantities are defined as

$$
\begin{gathered}
\omega_{0}^{2}=\frac{\left(4 \mu-a c_{s}^{2} \rho_{s}-P_{l 0}\right) R_{\mathrm{be}}-2 \sigma_{l} R_{\mathrm{ce}}^{3} / R_{\mathrm{be}}^{3}}{\beta \rho_{l} R_{\mathrm{ce}}^{2}\left(R_{\mathrm{ce}}-R_{\mathrm{be}}\right)} \\
\alpha=\frac{1}{2 \beta \rho_{l}}\left[\frac{4 \eta_{l}}{R_{\mathrm{be}}^{2}}\left(1+\frac{R_{\mathrm{be}}}{R_{\mathrm{ce}}}+\frac{R_{\mathrm{be}}^{2}}{R_{\mathrm{ce}}^{2}}\right)+\frac{\rho_{s} R_{\mathrm{be}}^{2}\left(4 \mu-a c_{s}^{2} \rho_{s}-P_{l 0}-2 \sigma_{l} R_{\mathrm{ce}}^{3} / R_{\mathrm{be}}^{4}\right)}{c_{s} \rho_{l} R_{\mathrm{ce}}\left(R_{\mathrm{ce}}-R_{\mathrm{be}}\right)\left[R_{\mathrm{ce}}-R_{\mathrm{be}}\left(1-\rho_{s} / \rho_{l}\right)\right]}\right] \\
\beta=1+\frac{\rho_{s} R_{\mathrm{be}}}{\rho_{l}\left(R_{\mathrm{ce}}-R_{\mathrm{be}}\right)}+\frac{4 \eta_{l} \rho_{s}\left(R_{\mathrm{ce}}^{2}+R_{\mathrm{ce}} R_{\mathrm{be}}+R_{\mathrm{be}}^{2}\right)}{c_{s} \rho_{l}^{2} R_{\mathrm{be}} R_{\mathrm{ce}}\left[R_{\mathrm{ce}}-R_{\mathrm{be}}\left(1-\rho_{s} / \rho_{l}\right)\right]} .
\end{gathered}
$$

In these equations, for simplicity, we have set $R_{c 0}=R_{\text {ce }}$ as these values are very close.

A solution to Eq. (66) is given by

$$
\omega=-i \alpha+\omega_{0} \sqrt{1-\alpha^{2} / \omega_{0}^{2}}
$$

The natural frequency of the bubble oscillation is defined as $f_{0}=\operatorname{Re}[\omega] / 2 \pi$ and the attenuation coefficient is equal to $\alpha$.

Figure 2 compares the results given by the linearized equations derived in the present paper with the predictions of the linear theory developed by Drysdale et al. [25]; see Sec. IIF2 in their paper. The calculations were performed at the following values of the physical parameters: $\rho_{l}=998 \mathrm{~kg} / \mathrm{m}^{3}, c=1484 \mathrm{~m} / \mathrm{s}, \quad \eta_{l}=0.001 \mathrm{~Pa} \mathrm{~s}$,
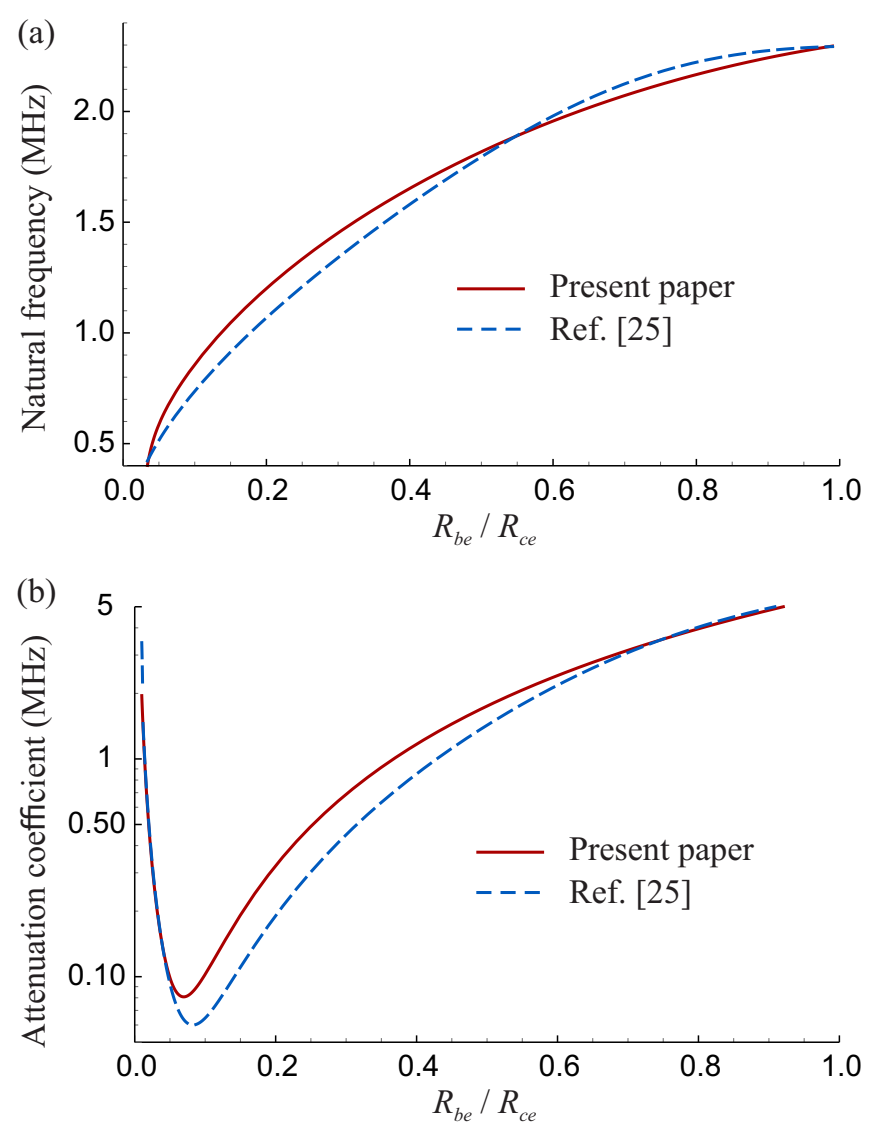

FIG. 2. Comparison of the linearized equations derived in the present paper with the linear theory developed by Drysdale et al. [25]. $\sigma_{l}=0.0725 \mathrm{~N} / \mathrm{m}, \quad \rho_{s}=1233 \mathrm{~kg} / \mathrm{m}^{3}, \quad \mu=0.74 \mathrm{GPa}, \quad c_{s}=$ $2111 \mathrm{~m} / \mathrm{s}, \quad P_{\infty}=101.3 \mathrm{kPa}, \quad P_{l 0}=-20 \mathrm{MPa}$, and $R_{\mathrm{ce}}=$ $100 \mu \mathrm{m}$. The parameters of the liquid correspond to water. The parameters of the solid medium were adopted from the paper of Drysdale et al. [25]. They correspond to cavitation experiments on transparent biomimetic wood [15-17].

Figures 2(a) and 2(b) show, respectively, the natural frequency and the attenuation coefficient as functions of the ratio $R_{\text {be }} / R_{\text {ce }}$. The solid lines show the results of the present paper and the dashed lines represent the results given by the theory of Drysdale et al. [25]. The difference between the curves is mainly a consequence of the fact that the models have a different precision with respect to the liquid compressibility. The equations derived in the present paper are correct up to the order $1 / c$, while the equations of Drysdale et al. [25] involve terms of the order $1 / c^{2}$. If these terms are omitted, the difference between the curves becomes insignificant.

\section{NUMERICAL SIMULATIONS}

Simulations were carried out at the values of the physical parameters that are indicated in Sec. II C 3. It was assumed that there was vacuum inside the bubble $\left(P_{b}=0\right)$.

Figure 3(a) shows the growth and the subsequent damped oscillation of the bubble, Fig. 3(b) shows the oscillation of the cavity surface, and Fig. 3(c) allows one to visually compare the oscillation amplitudes of the bubble and the cavity surface. In this simulation, $R_{c 0}=100 \mu \mathrm{m}$ and $P_{l 0}=-20 \mathrm{MPa}$. The initial bubble radius $R_{b 0}$, which corresponds to this value of $P_{l 0}$, is $7.25 \mathrm{~nm}$. The initial velocities of the bubble and the cavity surfaces were set equal to zero, $\dot{R}_{b}(0)=\dot{R}_{c}(0)=0$.

As expected, the oscillation of the solid surface is much weaker than the bubble oscillation, and its amplitude is very small compared to $R_{c 0}$. The calculations show that the final equilibrium radii of the bubble and the cavity are $R_{\mathrm{be}}=$ $27.37 \mu \mathrm{m}$ and $R_{\mathrm{ce}}=100.675 \mu \mathrm{m}$. The same values are given by Eqs. (62) and (63). By fitting experimental data, Vincent et al. [16] have come to the conclusion that $R_{\mathrm{be}} / R_{\mathrm{ce}} \approx 0.28$. Wang [24] argues that a more accurate fitting gives 0.265 . As one can see, our result, $R_{\mathrm{be}} / R_{\mathrm{ce}} \approx 0.27$, is in good agreement with these estimations. The approximation of the bubble attenuation by an exponential dependence, shown in Fig. 3(a) by the dashed line, indicates that the attenuation coefficient $\alpha$ is of the order of $0.5 \mathrm{MHz}$.

Figure 4 shows the normalized Fourier spectrum of the bubble oscillation depicted in Fig. 3(a), whence it follows that the natural frequency of the bubble oscillation is $f_{0}=1.36 \mathrm{MHz}$. This value is also in agreement with 

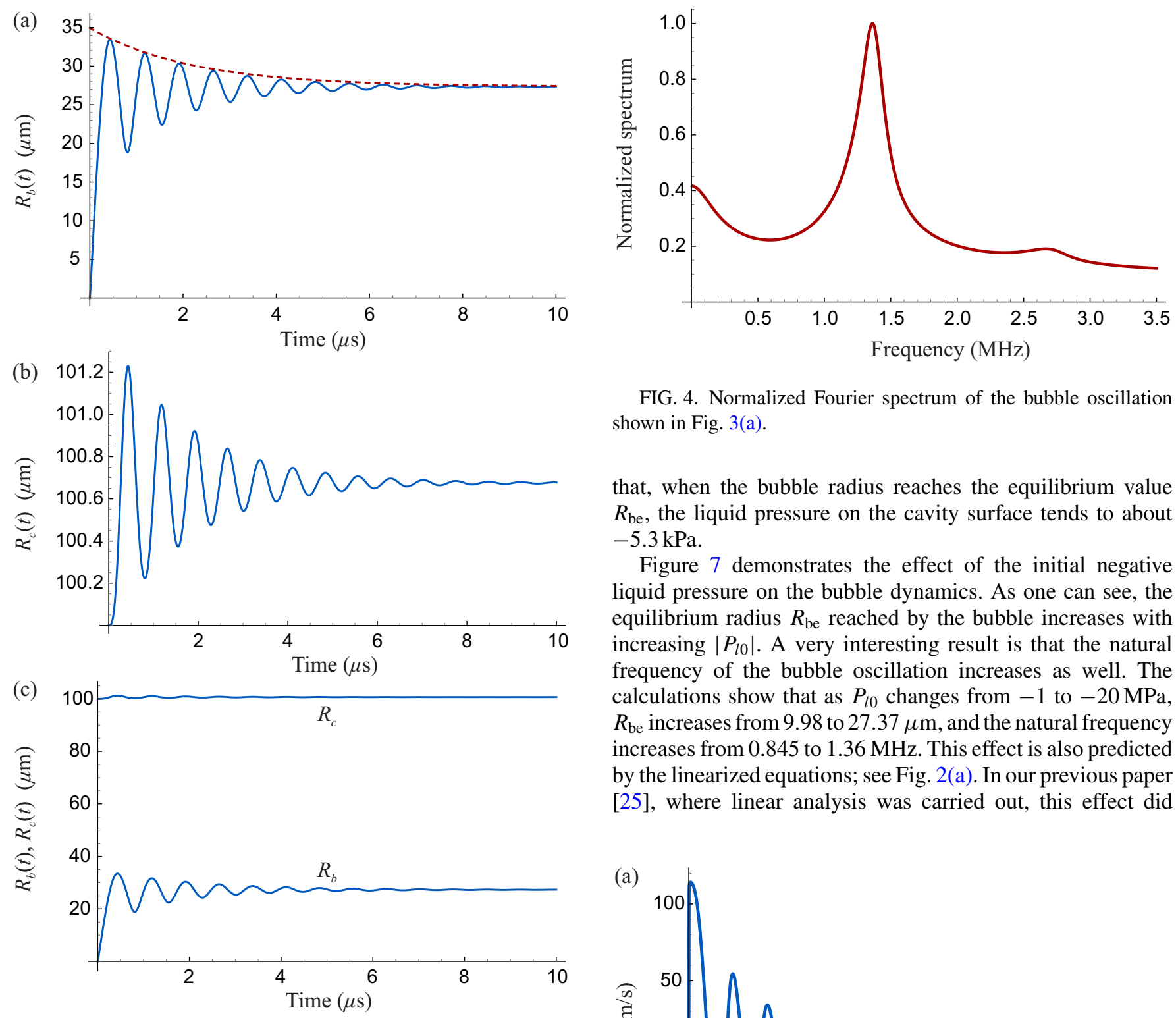

FIG. 4. Normalized Fourier spectrum of the bubble oscillation shown in Fig. 3(a).

that, when the bubble radius reaches the equilibrium value $R_{\mathrm{be}}$, the liquid pressure on the cavity surface tends to about $-5.3 \mathrm{kPa}$.

Figure 7 demonstrates the effect of the initial negative liquid pressure on the bubble dynamics. As one can see, the equilibrium radius $R_{\text {be }}$ reached by the bubble increases with increasing $\left|P_{l 0}\right|$. A very interesting result is that the natural frequency of the bubble oscillation increases as well. The calculations show that as $P_{l 0}$ changes from -1 to $-20 \mathrm{MPa}$, $R_{\text {be }}$ increases from 9.98 to $27.37 \mu \mathrm{m}$, and the natural frequency increases from 0.845 to $1.36 \mathrm{MHz}$. This effect is also predicted by the linearized equations; see Fig. 2(a). In our previous paper [25], where linear analysis was carried out, this effect did

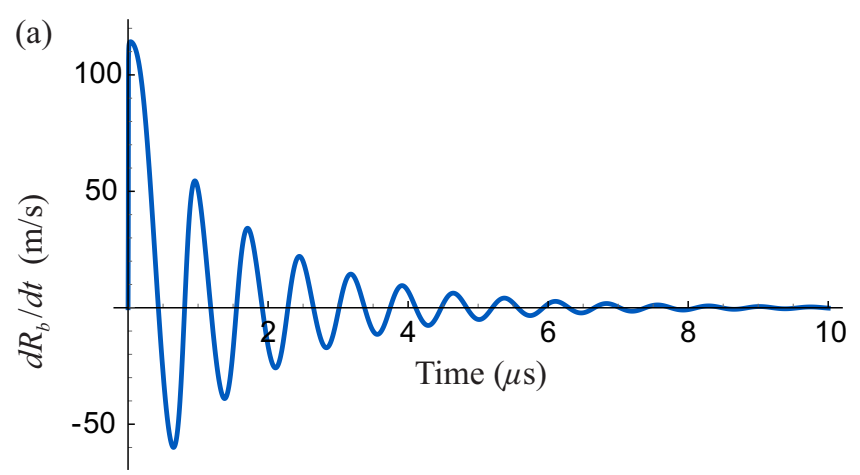

experimental measurements reported by Vincent et al. [16]. The quality factor of the bubble oscillation given by the above values of the natural frequency and the attenuation coefficient is $Q=\pi f_{0} / \alpha=8.5$. The resonance peak shown in Fig. 4 is rather wide. Note also the development of the second harmonic. These signs indicate that the bubble oscillation is not perfectly harmonic.

Figure 5 allows one to compare the velocity of the bubble surface with that of the cavity surface. As one can see, the former is much higher than the latter, but both of them are much smaller than the speeds of sound in the liquid and in the solid, $c$ and $c_{s}$. It should be also mentioned that the value of $\dot{R}_{b}$ is in agreement with evaluations made by Vincent $e t$ al. [15].

Figure 6 shows the evolution of the normal stress on the cavity surface, calculated by Eq. (A12). The calculations show

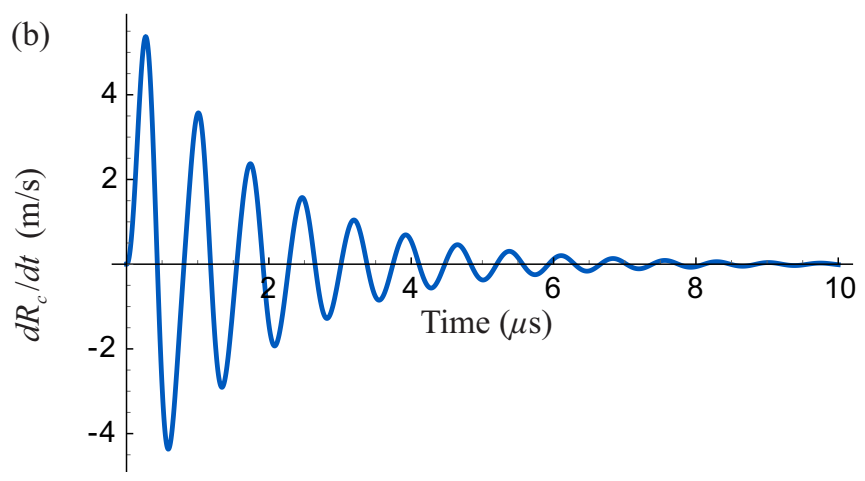

FIG. 5. (a) Velocity of the bubble surface. (b) Velocity of the cavity surface. 


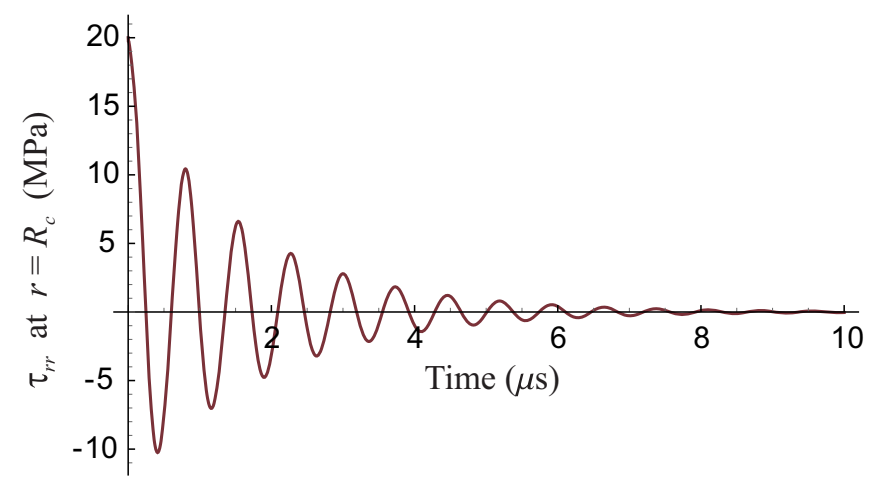

FIG. 6. Normal stress at the cavity surface.

not receive a proper attention. Therefore, we would like to emphasize it now. The prediction that the natural frequency increases with increasing bubble radius is in contrast with the bubble behavior in an unbounded liquid, where the natural frequency of a bubble is known to decrease with increasing bubble radius [1,2]. However, the agreement of this prediction with the experimental data of Vincent et al. [16] indicates the reality of this effect. An explanation is that the dynamics of the system under consideration is governed by the solid environment rather than by the bubble. If we look at Eq. (67), we will see that it predicts that, first, the resonance properties of the system are governed by the elastic properties of the solid environment. Second, the natural frequency should increase with increasing $R_{\text {be }}$ and decreasing the thickness of the liquid layer between the bubble and the cavity surface. For these reasons, even a vacuum bubble can resonate in the system under consideration, whereas in an unbounded liquid, that is impossible.

Figure 8 illustrates the bubble behavior at different values of the shear modulus $\mu$. In real solids, a change in $\mu$ means changes in other parameters as well. Therefore, to bring our analysis closer to reality, when we change $\mu$, we keep constant the solid density $\rho_{s}$ and Poisson's ratio but change the longitudinal wave speed $c_{s}$ and the Lamé coefficient $\lambda$

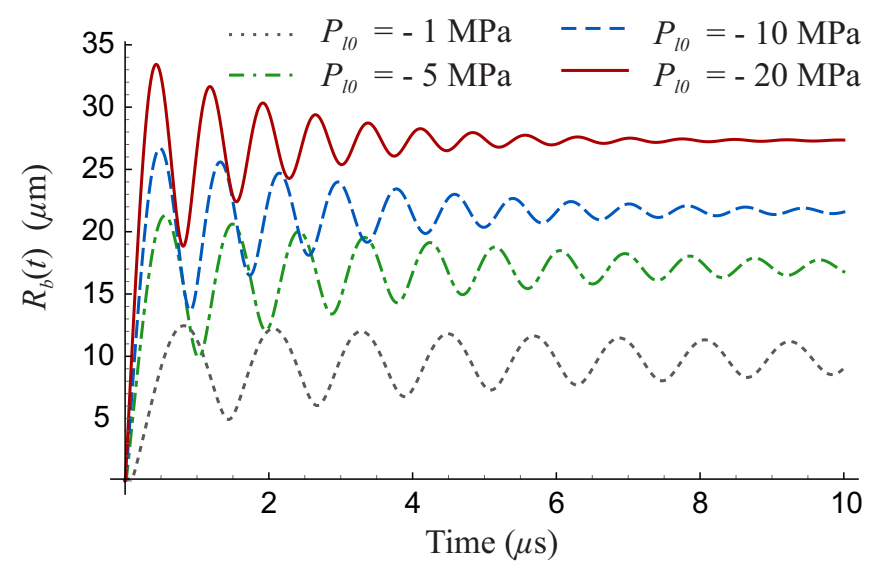

FIG. 7. Bubble growth and oscillation at different values of the initial negative liquid pressure.

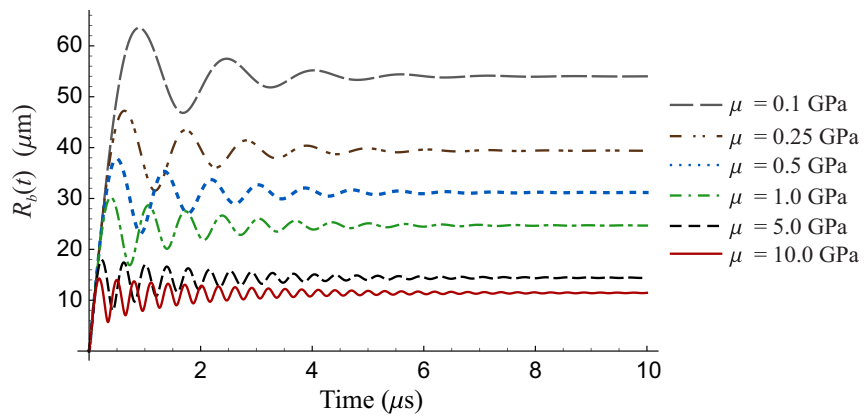

FIG. 8. Bubble growth and oscillation at different values of the shear modulus $\mu$.

according to the known formulas [27]:

$$
c_{s}=\sqrt{\frac{2 \mu(1-\nu)}{\rho_{s}(1-2 \nu)}}, \quad \lambda=c_{s}^{2} \rho_{s}-2 \mu,
$$

where $v$ is Poisson's ratio. In our previous simulations, $v=$ 0.4 . We keep the same value in the simulations with varying $\mu$. The value of $\rho_{s}$ is also kept as above, $\rho_{s}=1233 \mathrm{~kg} / \mathrm{m}^{3}$. Figure 8 shows that with increasing $\mu, R_{\text {be }}$ decreases and the natural frequency increases. The calculations show that as $\mu$ increases from 0.1 to $10 \mathrm{GPa}, R_{\text {be }}$ decreases from 54.01 to $11.43 \mu \mathrm{m}$, while the natural frequency increases from 0.635 to $3.33 \mathrm{MHz}$. In this case, the behavior of the natural frequency is expectable, considering its determining dependence on $\mu$ [see Eq. (67)], as a result of which the effect of increasing $\mu$ on the natural frequency is the deciding factor.

When we change $\mu$, we change the specific acoustic impedance of the solid, $z_{s}=\rho_{s} c_{s}$, so we can examine how a change in $z_{s}$ affects the attenuation coefficient $\alpha$. The dependence of $\alpha$ on $z_{s}$ is presented in Fig. 9. As one can see, the damping of the bubble oscillation monotonically decreases as the rigidity of the elastic medium increases. In our case, the specific acoustic impedance of the liquid (water) is $z_{l}=\rho_{l} c=1.48 \mathrm{MPa} \mathrm{s} / \mathrm{m}$. It is interesting to note that Fig. 9 does not reveal any singularities when $z_{s}$ passes through the

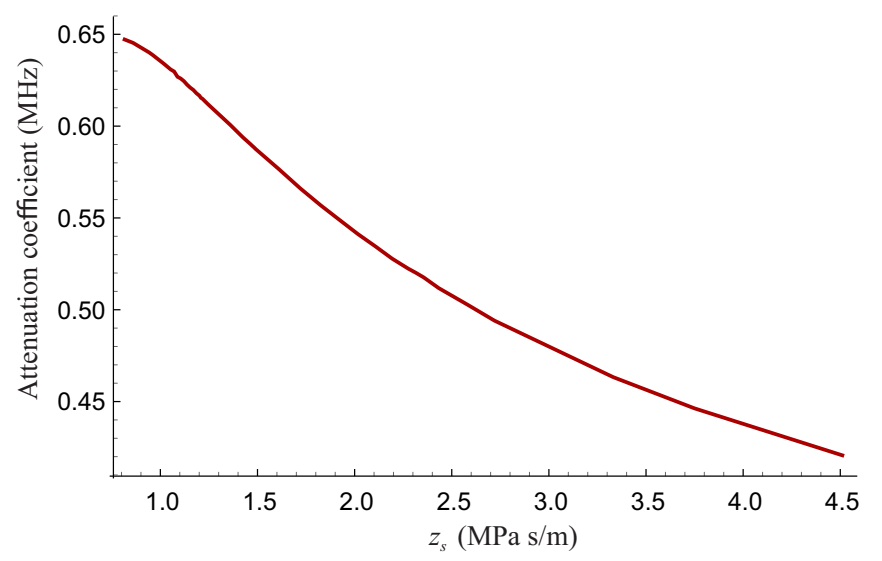

FIG. 9. Dependence of the attenuation coefficient $\alpha$ on the specific acoustic impedance of the solid $z_{s}=\rho_{s} c_{s}$. The physical parameters are as in Fig. 8. The longitudinal wave speed $c_{s}$ is calculated by Eq. (71) varying the value of $\mu$. 
value of $z_{l}$, i.e., the matching of the acoustic impedances of the liquid and the solid appears not to lead to a maximization of attenuation.

\section{CONCLUSION}

In the present paper, equations have been derived that model the growth and subsequent damped oscillation of a cavitation bubble in a liquid-filled cavity surrounded by an elastic solid. It was assumed that the nucleation and the growth of the bubble were caused by an initial negative pressure in the cavity. The liquid was treated as viscous and compressible. The obtained equations generalize the Keller-Miksis theory, which was derived for finite-amplitude oscillations of a bubble in an unbounded compressible liquid. Numerical simulations have shown that the final equilibrium radius reached by the growing bubble decreases when the absolute magnitude of the initial negative pressure decreases. It was also shown that the natural frequency of the bubble oscillation decreased with decreasing bubble radius, in contrast to what occurs in an unbounded liquid, where the natural frequency of a bubble increases with decreasing bubble radius.

\section{ACKNOWLEDGMENT}

This research has received funding from the European Research Council under the European Union's Seventh Framework Programme (FP7/2007-2013)/ERC Grant Agreement No. 614655 "Bubbleboost."

\section{APPENDIX A: CALCULATION OF $\tau_{r r}\left(R_{c}, t\right)$}

Let us first express $\tau_{r r}\left(R_{c}, t\right)$, given by Eq. (15), in terms of $s^{\prime}\left(t-R_{c} / c_{s}\right)$ only. Substitution of Eq. (13) into Eq. (19) yields

$$
s^{\prime}\left(t-R_{c} / c_{s}\right)+\frac{R_{c}}{c_{s}} s^{\prime \prime}\left(t-R_{c} / c_{s}\right)=R_{c}^{2} \dot{R}_{c} .
$$

This equation allows one to express $s^{\prime \prime}\left(t-R_{c} / c_{s}\right)$ in terms of $s^{\prime}\left(t-R_{c} / c_{s}\right)$

$$
\frac{R_{c}}{c_{s}} s^{\prime \prime}\left(t-R_{c} / c_{s}\right)=R_{c}^{2} \dot{R}_{c}-s^{\prime}\left(t-R_{c} / c_{s}\right) .
$$

Equation (13) allows one to express $s\left(t-R_{c} / c_{s}\right)$ in terms of $s^{\prime}\left(t-R_{c} / c_{s}\right)$ as

$$
s\left(t-R_{c} / c_{s}\right)=R_{c}^{2} u\left(R_{c}, t\right)-\frac{a R_{c}^{3}}{3}-\frac{R_{c}}{c_{s}} s^{\prime}\left(t-R_{c} / c_{s}\right) .
$$

Equations (A2) and (A3) allow one to express $\tau_{r r}\left(R_{c}, t\right)$ in terms of $s^{\prime}\left(t-R_{c} / c_{s}\right)$ only. However, before doing so, we need to calculate $u\left(R_{c}, t\right)$.

The displacement of the cavity surface $u\left(R_{c}, t\right)$ is defined by

$$
u\left(R_{c}, t\right)=R_{c}-\bar{R}_{c},
$$

where $\bar{R}_{c}$ denotes the unstrained position of the cavity surface. At $t=0$, Eq. (A4) becomes

$$
u\left(R_{c 0}, 0\right)=R_{c 0}-\bar{R}_{c}
$$

where $R_{c 0}=R_{c}(0)$. Eliminating $\bar{R}_{c}$ from Eq. (A4) by Eq. (A5), one obtains

$$
u\left(R_{c}, t\right)=R_{c}-R_{c 0}+u\left(R_{c 0}, 0\right) .
$$

The value of $u\left(R_{c 0}, 0\right)$ can be calculated by using the results of Task 2 of Sec. 7 in the book of Landau and Lifshitz [27]. Applying these results to our case, we get

$$
u\left(R_{c 0}, 0\right)=\frac{R_{c 0}}{4 \mu}\left[P_{l 0}+(\lambda+2 \mu) a\right],
$$

where $P_{l 0}$ denotes the initial (negative) pressure in the liquid.

Setting $r=R_{c}$ in Eq. (15) and substituting Eqs. (A2), (A3), (A6), and (A7) into it, one obtains

$$
\begin{aligned}
\tau_{r r}\left(R_{c}, t\right)= & {[(\lambda+2 \mu) a-4 \mu]\left(1-\frac{R_{c 0}}{R_{c}}\right)-\frac{R_{c 0} P_{l 0}}{R_{c}} } \\
& +\frac{\lambda+2 \mu}{c_{s} R_{c}^{2}}\left[s^{\prime}\left(t-R_{c} / c_{s}\right)-R_{c}^{2} \dot{R}_{c}\right] .
\end{aligned}
$$

We have not imposed so far any restrictions on time delays in the elastic solution. However, to proceed, we have to assume that the effect of the time delay $R_{c} / c_{s}$ on the behavior of the function $s\left(t-R_{c} / c_{s}\right)$ and its derivatives is small and hence all these functions can be expanded into a Taylor series in terms of $R_{c} / c_{s}$. With this assumption, we get

$$
s^{\prime}\left(t-R_{c} / c_{s}\right)=s^{\prime}(t)-\frac{R_{c}}{c_{s}} s^{\prime \prime}(t)+\frac{R_{c}^{2}}{2 c_{s}^{2}} s^{\prime \prime \prime}(t)+O\left(\frac{1}{c_{s}^{3}}\right) .
$$

It follows from expanding Eq. (A1) into a similar Taylor series that

$$
s^{\prime}(t)=R_{c}^{2} \dot{R}_{c}+\frac{R_{c}^{2}}{2 c_{s}^{2}} s^{\prime \prime \prime}(t)+O\left(\frac{1}{c_{s}^{3}}\right) .
$$

Substituting Eq. (A10) into Eq. (A9) and keeping terms up to the order $1 / c_{s}^{2}$, we obtain

$$
\begin{aligned}
& s^{\prime}\left(t-R_{c} / c_{s}\right)-R_{c}^{2} \dot{R}_{c} \\
& =-\frac{R_{c}}{c_{s}} \frac{d}{d t}\left(R_{c}^{2} \dot{R}_{c}\right)+\frac{R_{c}^{2}}{c_{s}^{2}} \frac{d^{2}}{d t^{2}}\left(R_{c}^{2} \dot{R}_{c}\right)+O\left(\frac{1}{c_{s}^{3}}\right) .
\end{aligned}
$$

Substitution of Eq. (A11) into Eq. (A8) yields

$$
\tau_{r r}\left(R_{c}, t\right)=\tau_{0}(t)+\tau_{2}(t)+\tau_{3}(t)+O\left(\frac{1}{c_{s}^{4}}\right),
$$

where the subscript shows the order of the terms in $1 / c_{s}$, so that

$$
\begin{gathered}
\tau_{0}(t)=[(\lambda+2 \mu) a-4 \mu]\left(1-\frac{R_{c 0}}{R_{c}}\right)-\frac{R_{c 0} P_{l 0}}{R_{c}}, \\
\tau_{2}(t)=-\frac{\lambda+2 \mu}{c_{s}^{2} R_{c}} \frac{d}{d t}\left(R_{c}^{2} \dot{R}_{c}\right)=-\frac{\lambda+2 \mu}{c_{s}^{2}}\left(R_{c} \ddot{R}_{c}+2 \dot{R}_{c}^{2}\right),
\end{gathered}
$$

$$
\begin{aligned}
\tau_{3}(t) & =\frac{\lambda+2 \mu}{c_{s}^{3}} \frac{d^{2}}{d t^{2}}\left(R_{c}^{2} \dot{R}_{c}\right) \\
& =\frac{\lambda+2 \mu}{c_{s}^{3}}\left(R_{c}^{2} \dddot{R}_{c}+6 R_{c} \dot{R}_{c} \ddot{R}_{c}+2 \dot{R}_{c}^{3}\right)
\end{aligned}
$$


The problem is the appearance of the third derivative in Eq. (A15). It can be resolved as follows.

The factor $1 / c_{s}^{3}$ in Eq. (A15) allows us to use solutions of lower accuracy to eliminate $\dddot{R}_{c}$, namely, Eqs. (28) and (29) for an incompressible liquid. From Eq. (29), $\dddot{R}_{c}$ can be expressed in terms of $\dddot{R}_{b}$. Then, by differentiating Eq. (28), $\dddot{R}_{b}$ is expressed in terms of derivatives of lower order, keeping in $\tau_{r r}\left(R_{c}, t\right)$ on the right-hand side of Eq. (28) only the terms $\tau_{0}$ and $\tau_{2}$. The calculation can be simplified considering that the velocity of the elastic oscillation is much smaller than the velocity of the bubble oscillation, i.e., $\dot{R}_{c} \ll \dot{R}_{b}$. Numerical results presented in Sec. III confirm this expected fact. Therefore, without an essential loss in accuracy, $R_{c}$ can be considered as a constant when the left-hand side of Eq. (28) is differentiated. The final result of this calculation is

$$
\begin{aligned}
& R_{c}^{2} \ddot{R}_{c}\left(1-\frac{R_{b}}{R_{c}}+\frac{\rho_{s} R_{b}}{\rho_{l} R_{c}}\right) \\
& =\left(a c_{s}^{2} \rho_{s}+P_{l 0}-4 \mu\right) \frac{R_{b} R_{c 0} \dot{R}_{c}}{\rho_{l} R_{c}^{2}}+\frac{2 \sigma_{l} \dot{R}_{b}}{\rho_{l} R_{b}}-\frac{4 \eta_{l} \ddot{R}_{b}}{\rho_{l}}\left(1-\frac{R_{b}^{3}}{R_{c}^{3}}\right)+\frac{R_{b} \dot{P}_{b}}{\rho_{l}}-R_{c} \dot{R}_{c} \ddot{R}_{c}\left(6-\frac{6 R_{b}}{R_{c}}+\frac{5 R_{b} \rho_{s}}{R_{c} \rho_{l}}\right) \\
& \quad+R_{b} \dot{R}_{b} \ddot{R}_{b}\left(2-\frac{R_{b}^{4}}{R_{c}^{4}}\right)+\frac{4 \eta_{l}}{\rho_{l}} \frac{\dot{R}_{b}^{2}}{R_{b}}\left(1+\frac{2 R_{b}^{3}}{R_{c}^{3}}\right)-2 \dot{R}_{c}^{3}\left(1-\frac{R_{b}}{R_{c}}\right)+2 \dot{R}_{b}^{3}\left(1-\frac{R_{b}^{4}}{R_{c}^{4}}\right) .
\end{aligned}
$$

Here, we have also used Eq. (10).

Equation (A16) makes it possible to eliminate $\dddot{R}_{c}$ from Eq. (A15). However, it is very cumbersome and in fact superfluous. The point is that the magnitude of the elastic oscillation is very small compared to the initial radius of the cavity, $R_{c 0}$, and its final equilibrium radius, $R_{\mathrm{ce}}$. Therefore, Eq. (A16) can be linearized with respect to time derivatives. This yields

$$
R_{c}^{2} \dddot{R}_{c}\left(1-\frac{R_{b}}{R_{c}}+\frac{\rho_{s} R_{b}}{\rho_{l} R_{c}}\right)=\left(a c_{s}^{2} \rho_{s}+P_{l 0}-4 \mu\right) \frac{R_{b} R_{c 0} \dot{R}_{c}}{\rho_{l} R_{c}^{2}}+\frac{2 \sigma_{l} R_{c}^{2} \dot{R}_{c}}{\rho_{l} R_{b}^{3}}-\frac{4 \eta_{l}\left(R_{c}^{3}-R_{b}^{3}\right) \ddot{R}_{c}}{\rho_{l} R_{b}^{2} R_{c}}+\frac{R_{b} \dot{P}_{b}}{\rho_{l}} .
$$

Here, we have also used Eq. (29) to replace the derivatives of $R_{b}$ with those of $R_{c}$. With Eq. (A17), using also Eq. (10), we can recast Eq. (A15) to

$$
\tau_{3}(t)=\frac{\rho_{s}}{c_{s} \rho_{l}\left[R_{c}-R_{b}\left(1-\rho_{s} / \rho_{l}\right)\right]}\left[\left(a c_{s}^{2} \rho_{s}+P_{l 0}-4 \mu\right) \frac{R_{b} R_{c 0} \dot{R}_{c}}{R_{c}}+\frac{2 \sigma_{l} R_{c}^{3} \dot{R}_{c}}{R_{b}^{3}}-\frac{4 \eta_{l}\left(R_{c}^{3}-R_{b}^{3}\right) \ddot{R}_{c}}{R_{b}^{2}}+R_{b} R_{c} \dot{P}_{b}\right] .
$$

Since the value of $\mu$ is usually of the order of GPa, the behavior of Eq. (A18) is determined by the first term in brackets, while the other terms are only small corrections.

\section{APPENDIX B: EQUATIONS USED FOR THE DERIVATION OF EQ. (56)}

For $c \rightarrow \infty$, Eq. (52) reduces to

$$
E_{b}^{\mathrm{inc}}=P_{b}-\rho_{l} R_{b} \ddot{R}_{b}-\frac{3}{2} \rho_{l} \dot{R}_{b}^{2}-\frac{4 \eta_{l} \dot{R}_{b}}{R_{b}}-\frac{2 \sigma_{l}}{R_{b}} .
$$

Differentiating Eq. (B1) gives

$$
\dot{E}_{b}^{\mathrm{inc}}=\dot{P}_{b}-\rho_{l}\left(R_{b} \dddot{R}_{b}+\dot{R}_{b} \ddot{R}_{b}\right)-3 \rho_{l} \dot{R}_{b} \ddot{R}_{b}-4 \eta_{l}\left(\frac{\ddot{R}_{b}}{R_{b}}-\frac{\dot{R}_{b}^{2}}{R_{b}^{2}}\right)+\frac{2 \sigma_{l} \dot{R}_{b}}{R_{b}^{2}} .
$$

To eliminate $\dddot{R}_{b}$, Eq. (28) is used. Its differentiation gives

$$
\begin{aligned}
\rho_{l}\left(R_{b} \dddot{R}_{b}+\dot{R}_{b} \ddot{R}_{b}\right)= & \frac{R_{c}}{R_{c}-R_{b}}\left\{\dot{P}_{b}+\frac{d \tau_{r r}\left(R_{c}, t\right)}{d t}+\frac{2 \dot{R}_{b} \sigma_{l}}{R_{b}^{2}}-\frac{12 \eta_{l} R_{b}^{2} \dot{R}_{b} \dot{R}_{c}}{R_{c}^{4}}-\rho_{l} \ddot{R}_{b}\left[3 \dot{R}_{b}-\frac{5 R_{b} \dot{R}_{b}}{R_{c}}+\frac{\dot{R}_{b} R_{b}^{4}}{R_{c}^{4}}+\frac{R_{b}^{2} \dot{R}_{c}}{R_{c}^{2}}\right.\right. \\
& \left.\left.+\frac{4 \eta_{l}}{\rho_{l} R_{b}}\left(1-\frac{R_{b}^{3}}{R_{c}^{3}}\right)\right]-\rho_{l} \dot{R}_{b}^{2}\left[\frac{2 R_{b}^{3} \dot{R}_{b}}{R_{c}^{4}}-\frac{2 \dot{R}_{b}}{R_{c}}+\frac{2 R_{b} \dot{R}_{c}}{R_{c}^{2}}-\frac{2 R_{b}^{4} \dot{R}_{c}}{R_{c}^{5}}-\frac{4 \eta_{l}}{\rho_{l} R_{b}^{2}}\left(1+\frac{2 R_{b}^{3}}{R_{c}^{3}}\right)\right]\right\} .
\end{aligned}
$$

Substitution of Eq. (B3) into Eq. (B2) yields

$$
\begin{aligned}
\dot{E}_{b}^{\text {inc }}= & \frac{1}{R_{c}-R_{b}}\left\{-R_{b} \dot{P}_{b}-R_{c} \frac{d \tau_{r r}\left(R_{c}, t\right)}{d t}-\frac{2 \sigma_{l} \dot{R}_{b}}{R_{b}}+\frac{12 \eta_{l} R_{b}^{2} \dot{R}_{b} \dot{R}_{c}}{R_{c}^{3}}+\rho_{l} \ddot{R}_{b}\left[R_{b} \dot{R}_{b}\left(\frac{R_{b}^{3}}{R_{c}^{3}}-2\right)\right.\right. \\
& \left.\left.+\frac{R_{b}^{2} \dot{R}_{c}}{R_{c}}+\frac{4 \eta_{l}}{\rho_{l}}\left(1-\frac{R_{b}^{2}}{R_{c}^{2}}\right)\right]+\rho_{l} \dot{R}_{b}^{2}\left[2 \dot{R}_{b}\left(\frac{R_{b}^{3}}{R_{c}^{3}}-1\right)+\frac{2 \dot{R}_{c} R_{b}}{R_{c}}\left(1-\frac{R_{b}^{3}}{R_{c}^{3}}\right)-\frac{4 \eta_{l}}{\rho_{l} R_{b}}\left(1+\frac{2 R_{b}^{2}}{R_{c}^{2}}\right)\right]\right\} .
\end{aligned}
$$

Substitution of Eq. (B4) along with Eqs. (52) and (53) into Eq. (55) results in Eq. (56). 
[1] T. G. Leighton, The Acoustic Bubble (Academic Press, London, 1994).

[2] C. E. Brennen, Cavitation and Bubble Dynamics (Oxford University Press, New York, 1995).

[3] Lord Rayleigh, Phil. Mag. 34, 94 (1917).

[4] C. Herring, OSRD Report No. 236 (1941).

[5] R. H. Cole, Underwater Explosions (Princeton University Press, Princeton, NJ, 1948), pp. 305-307.

[6] M. S. Plesset, J. Appl. Mech. 16, 277 (1949).

[7] L. Trilling, J. Appl. Phys. 23, 14 (1952).

[8] F. R. Gilmore, Hydrodynamics Lab. Rep. No. 26-4 (California Institute of Technology, Pasadena, CA, 1952).

[9] J. B. Keller and I. I. Kolodner, J. Appl. Phys. 27, 1152 (1956).

[10] J. B. Keller and M. Miksis, J. Acoust. Soc. Am. 68, 628 (1980).

[11] H. Cochard, C. R. Phys. 7, 1018 (2006).

[12] A. D. Stroock, V. V. Pagay, M A. Zwieniecki, and N. M. Holbrook, Annu. Rev. Fluid Mech. 46, 615 (2014).

[13] M. Larter, T. J. Brodribb, S. Pfautsch, R. Burlett, and H. Cochard, Plant Physiol. 168, 804 (2015).

[14] K. H. Jensen, K. Berg-Sørensen, H. Bruus, N. M. Holbrook, J. Liesche, A. Schulz, M. A. Zwieniecki, and T. Bohr, Rev. Mod. Phys. 88, 035007 (2016).
[15] O. Vincent, P. Marmottant, P. A. Quinto-Su, and C.-D. Ohl, Phys. Rev. Lett. 108, 184502 (2012).

[16] O. Vincent, P. Marmottant, S. R. Gonzalez-Avila, K. Ando, and C.-D. Ohl, Soft Matter 10, 1455 (2014).

[17] O. Vincent and P. Marmottant, J. Fluid Mech. 827, 194 (2017).

[18] M. T. Tyree and M. A. Dixon, Plant Physiol. 72, 1094 (1983).

[19] S. Mayr and S. Rosner, Tree Physiol. 31, 59 (2011).

[20] M. T. Tyree and J. S. Sperry, Annu. Rev. Plant Phys. Mol. Bio. 40, 19 (1989).

[21] T. J. Brodribb and H. Cochard, Plant Physiol. 149, 575 (2009).

[22] B. Choat et al., Nature 491, 752 (2012).

[23] X. Noblin, N. O. Rojas, J. Westbrook, C. Llorens, M. Argentina, and J. Dumais, Science 335, 1322 (2012).

[24] Q. X. Wang, Phys. Fluids 29, 072101 (2017).

[25] C. Drysdale, A. A. Doinikov, and P. Marmottant, Phys. Rev. E 95, 053104 (2017).

[26] L. D. Landau and E. M. Lifshitz, Fluid Mechanics (Pergamon Press, Oxford, 1987).

[27] L. D. Landau and E. M. Lifshitz, Theory of Elasticity (Pergamon Press, Oxford, 1970). 